\title{
Riqueza e abundância de abelhas Euglossina (Hymenoptera, Apidae) em fragmento de floresta, no município de Caracaraí, Roraima, Brasil
}

\author{
Silvio José Reis da Silva ${ }^{1,2}$, Alessandro Souza da Silva ${ }^{1} \&$ Juliane Patrícia Ribeiro \\ Pereira $^{2}$
}

1.Universidade Estadual de Roraima. Rua Sete de Setembro, 231 - Bairro Canarinho. Boa Vista, Roraima. 69306-530 E-mail: silviojosereisdasilva@gmail.com

2. Instituto de Amparo de Ciência, Tecnologia e Inovação de Roraima. Museu Integrado de Roraima. Laboratório de Entomologia. Av. Brigadeiro Eduardo Gomes s.n., Parque Anauá, Bairro Aeroporto, Boa Vista, Roraima CEP 69305010 .

Autor para correspondência: silviojosereisdasilva@gmail.com

Recebido em: 08/09/2014 Aceito em: 10/03/2015 Publicado online em PDF: 10/04/2015.

\section{RESUMO}

Riqueza e abundância de abelhas Euglossina (Hymenoptera, Apidae) em fragmento de floresta, no município de Caracaraí, Roraima, Brasil. As abelhas estão representadas por quase 30.000 espécies. Dentre os diversos grupos de abelhas, as Euglossina, têm despertado grande interesse dos pesquisadores devido a facilidade de captura e pelo seu uso como bioindicadoras de alteração ambiental. Este estudo teve por objetivos determinar a riqueza e abundância de abelhas Euglossina atraídas por substâncias aromáticas em um fragmento de floresta na região do Bem-Querer, município de Caracaraí, Roraima, Brasil. As coletas foram realizadas no período de março a setembro de 2012. As abelhas foram capturadas por meio de armadilhas construídas com garrafas PET, contendo iscas impregnadas com eugenol, salicilato de metila e óleo de eucalipto. Foram escolhidas três estações de coletas distantes 500 metros entre si, ao longo da estrada de acesso as corredeiras. Nos três pontos foram capturados 510 espécimes, sendo: 504 machos identificados, duas fêmeas e quatro indivíduos machos que não foram identificados. Foram coletados espécimes dos gêneros: Euglossa (20 spp.), Eulaema (04 spp.), Exaerete (03 spp.) e Eufriesea (01 sp.), em um total de 28 espécies de abelhas Euglossina identificadas. O gênero Euglossa foi o mais abundante com 338 espécimes identificados (67,06\%), seguido de Eulaema com 132 espécimes (26,19\%), Exaerete com 32 (6,35\%) e Eufriesea com dois espécimes (0,40\%). A substância mais atrativa foi o óleo de eucalipto com 356 espécimes atraídos (70,63\%), seguida do salicilato de metila com 121 espécimes (24,00\%) e o eugenol com 27 $(5,35 \%)$. Estes resultados indicam a região das corredeiras do Bem-Querer como uma das localidades com alta diversidade de Euglossina no estado de Roraima.

PALAVRAS-CHAVE: Biodiversidade; Conservação; Floresta Amazônica.

\begin{abstract}
Richness and Abundance of Euglossina bees (Hymenoptera: Apidae) in forest fragment from Caracaraí city, Roraima state, Brazil. The bees are represented by almost 30,000 species. Among the many groups of bees, the Euglossina, have aware the research attention by the facility of captures and its use as a bioindicators of environmental quality. The aim of this study was to determine the richness and adundace of bees Euglossina attracted for aromatic substances in a forest fragment in the Bem-Querer, municipality of Caracaraí, Roraima, Brazil. The collects were made of March to September 2012. The bees were captured with PET bottles traps, containing baits impregnated with eugenol, methyl salicylate and eucalyptus oil. Were chosen three collects stations distant 500 meters from each to other, along the rapids access road. In three stations, 510 specimens were captured being: 504 males identified two females and four male individuals that were not identified. It was collected species from the genera Euglossa (20 spp.), Eulaema (04 spp.), Exaerete (03 spp.), Eufriesea (01 sp.), a total of 28 identified species of Euglossina bees. The genera Euglossa was the most abundant with 338 specimens (67.06\%), followed by the Eulaema with 132 specimens (26.19\%), the genera Exaerete had $32(6.35 \%)$ and Eufriesea with two specimens (0.40\%). The substance more attractive was the eucalyptus oil with 356 specimens attracted $(70.63 \%)$, followed by the methyl salicylate with 121 specimens $(24.00 \%)$ and eugenol with $27(5.35 \%)$. These results shows the region of the Bem-Querer as one location with high diversity of Euglossina in Roraima State.
\end{abstract}

KEY WORDS: Amazon Forest; Biodiversity; Conservation. 


\section{INTRODUÇÃO}

As abelhas são um grupo de enorme importância para as plantas que necessitam de agentes polinizadores para produzirem frutos e sementes. Muitas plantas cultivadas só produzem frutos satisfatoriamente na presença das abelhas (Crane 1972; Roubik 1989; Silva 2010).

Dentre os diversos grupos de abelhas, as Euglossina são conhecidas como abelhas de orquídeas, pois são atraídas por substâncias aromáticas que estão presentes em suas flores. O processo de coleta de substâncias aromáticas é realizado apenas pelos machos que, ao coletarem essas substâncias, também contribuem para polinização das orquídeas, exercendo um importante papel ecológico (Peruquetti 2000). O fato de as abelhas Euglossina serem atraídas por substâncias aromáticas possibilita um método prático e eficiente de coleta desses insetos. A maioria dos estudos sobre diversidade de abelhas Euglossina utilizam substâncias aromáticas para atração de machos (Silveira et al. 2002; Oliveira et al. 2010). Dentre os vários compostos aromáticos, o óleo de eucalipto e o salicilato de metila foram considerados os mais atrativos em diversos estudos realizados em Roraima (Silva 2002; Maia \& Silva 2008; Silva et al. 2013).

As Euglossina são insetos robustos, com cores metálicas fortes e de vôo rápido. Sua distribuição é exclusivamente Neotropical e abrangem cerca de 200 espécies conhecidas, distribuídas em cinco gêneros: Eulaema, Euglossa, Eufriesea, Exaerete e Aglae sendo o último considerado não frequente (Tales et al. 2009; Silveira et al. 2002).

Essas abelhas também são consideradas bioindicadoras do estado de conservação natural, e são mais diversas em florestas úmidas como a Mata Atlântica e a Floresta Amazônica (Mendes et al. 2008; Ramalho et al. 2009; Nemésio 2013). Segundo Morato \& Alencar (2009), de modo geral, as áreas com predominância de vegetação natural ou conservada têm maior diversidade dessas abelhas, embora algumas espécies sejam coletadas em ambientes antropizados, urbanos e rurais.

Estudos em fragmentos florestais tem demonstrado que algumas espécies podem ser dependentes, não só do grau de alteração do ambiente, mas também de sua fragmentação já que podem forragear em uma extensa área. A fragmentação e a exploração dos remanescentes de mata nativa podem provocar a perda de várias espécies de Euglossina, indicando a necessidade de preservar esses remanescentes (Aguiar 2006). Por outro lado a capacidade dessas abelhas de voar longas distâncias (Janzen 1971; Roubik 1989, Wikelski et al. 2010) favorece sua distribuição homogênea nos fragmentos florestais, mesmo distantes alguns quilômetros uns dos outros. Mas segundo Becker et al. (1991), tanto a abundância quanto a riqueza de espécies de abelhas Euglossina podem variar numa dada localidade e na mesma época do ano, caso uma série de coletas e/ou armadilhas sejam delineadas a apenas algumas centenas de metros.

O Estado de Roraima reúne poucos trabalhos realizados com abelhas Euglossina. Neste estudo foram coligidos os trabalhos de Silva (2002) nas corredeiras do Bem-Querer, no Município de Caracaraí, Nemésio (2005) na Reserva Ecológica de Maracá (município de Amajarí), Maia \& Silva (2008) com abelhas Euglossina em seis localidades de florestas com plantios de acácia australiana (Acacia mangium Wild.), Oliveira et al. (2010) listando todas as espécies depositadas nas coleções do Instituto Nacional de Pesquisas da Amazônia e do Museu Integrado de Roraima e Silva et al. (2013) comparando a riqueza e abundância de Euglossina em área de floresta alterada e primária na Serra do Tepequém, município de Amajari.

Este estudo teve por objetivos determinar a riqueza e abundância de abelhas Euglossina atraídas por substâncias aromáticas em um fragmento de floresta na região do BemQuerer, município de Caracaraí, Roraima, Brasil.

\section{MATERIAL E MÉTODOS}

A pesquisa foi realizada em um fragmento de floresta primária preservada, com dimensões aproximadas de $3 \times 6 \mathrm{~km}$, localizado nas Corredeiras do Bem-Querer, Município de Caracaraí, Estado de Roraima, rodovia BR 174 no $\mathrm{Km} \mathrm{136,} \mathrm{no} \mathrm{sentido} \mathrm{Boa} \mathrm{Vista/Manaus}\left(1^{\circ}\right.$ $55^{\prime} 54^{\prime \prime} \mathrm{N}$ e $61^{\circ} 01^{\prime} 00^{\prime}$ ' W).

As coletas foram realizadas no período de março a setembro de 2012. Foram escolhidas três estações de coleta. As três estações distavam aproximadamente 500 metros entre si ao longo de um transecto de $1500 \mathrm{~m}$. Foi 
realizada uma coleta por mês, no horário de $07 \mathrm{~h}$ às $12 \mathrm{~h}$. Em cada estação foi montado um conjunto de três armadilhas, distantes dois metros uma da outra e a um metro e meio de altura do solo, formando um triângulo em cada estação de coleta. Uma armadilha continha uma isca impregnada com eugenol, outra com salicilato de metila e outra com óleo de eucalipto. A escolha dessas substâncias reside no fato de serem consideradas as mais atrativas e também pela facilidade de aquisição.

Cada armadilha foi construída com uma garrafa plástica PET de 2 litros de capacidade, a qual foi acoplada, em orifícios, os gargalos de três garrafas plásticas de $600 \mathrm{ml}$, sem o fundo e com uma camada de areia e cola na parte interna para auxiliar o acesso das abelhas para o interior. Cada garrafa possuía, em seu interior, um pedaço de tecido amarrado em uma haste de madeira que era impregnado com a substância aromática desejada. A extremidade oposta da haste de madeira foi fixada na tampa da garrafa. Essas armadilhas foram desenvolvidas pelo Laboratório de Entomologia do Museu Integrado de Roraima (Figura 01).

Após as coletas, os exemplares de abelhas Euglossina foram alfinetados, etiquetados e depositados na coleção do laboratório de Entomologia do Museu Integrado de Roraima MIRR. A identificação foi feita por comparação com a coleção de referência organizada pelo Dr. Márcio Luiz de Oliveira do Instituto Nacional de Pesquisas da Amazônia INPA. A grafia dos nomes científicos e a classificação taxonômica foram baseadas em Silveira et al. (2002) e Moure et al. (2015).

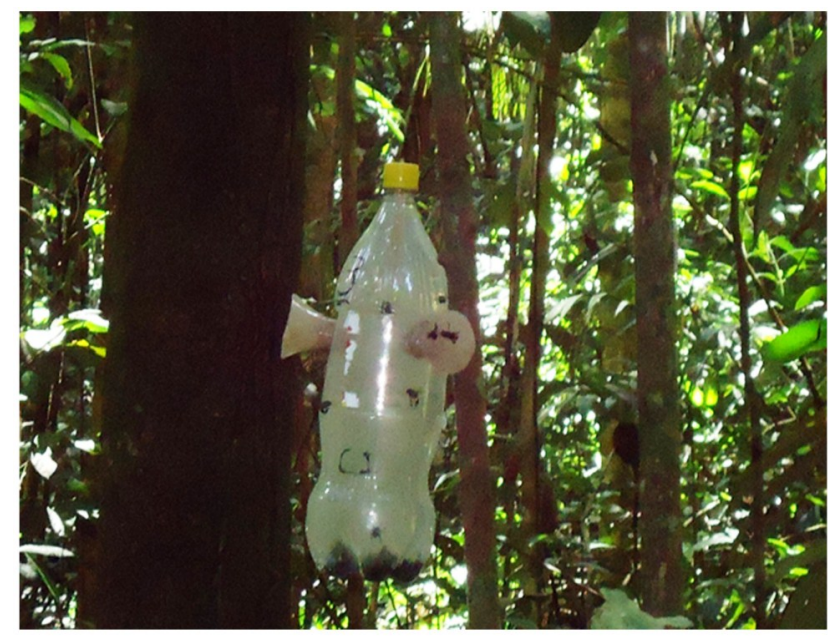

Figura 1. Armadilha construída de garrafa PET utilizada na captura das abelhas Euglossina.

\section{RESULTADOS E DISCUSSÃO}

Foram capturadas 28 espécies de Euglossina dos gêneros Eufriesea, Euglossa, Eulaema e Exaerete durante o período de coleta, em um total 510 indivíduos capturados, sendo 508 machos e duas fêmeas. Quatro indivíduos machos do gênero Euglossa não foram identificados por estarem com as estruturas das pernas médias bastante danificas impossibilitando assim, a visualização de caracteres de importância taxonômica. Esses indivíduos machos, bem como as duas fêmeas, foram excluídos da análise (Tabela 01). As fêmeas também foram excluídas da análise já que as chaves de identificação de espécies para o gênero Euglossa estão baseadas principalmente, nas estruturas das pernas medianas que são diferenciadas nos machos, o que impossibilita a identificação das fêmeas deste gênero.

Algumas espécies, como Euglossa orellana Roubik, 2004, Exaerete lepeletiere Oliveira \& Nemésio 2003 e Euglossa ioprosopa Dressler, 1982, não foram capturadas em trabalhos anteriores para a região do Bem-Querer.

Foram coletadas 20 espécies pertencentes ao gênero Euglossa que se mostrou como o gênero com maior riqueza específica na área de estudo $(71,43 \%)$, seguido por Eulaema com quatro espécies (14,29\%), Exaerete com três espécies $(10,71 \%)$ e Eufriesea com apenas uma espécie $(3,57 \%)$, de um total de 28 espécies capturadas e identificadas (Tabela 02).

Atualizando a lista de espécies dessas abelhas para as corredeiras do Bem-Querer e levando em consideração os dados contidos em Silva (2002) e Oliveira et al. (2010), a riqueza de espécies para essa localidade se eleva para 39 espécies.

O número de espécies de Euglossina, excluindo os espécimes não identificados, listado para este trabalho, é menor que o listada por Oliveira et al. (2010) com 36 espécies para a região das Corredeiras do Bem-Querer. Entretanto este valor foi muito próximo ao de outro trabalho realizado por Silva (2002), com 29 espécies, também realizadas nas corredeiras do Bem-Querer. Embora Oliveira et al. (2010) tenham citado 36 espécies de Euglossina para região do Bem-Querer, ao analisar a tabela correspondente, listamos apenas 34 espécies de abelhas desta grupo.

Estudos realizados em outros locais do estado por Maia \& Silva (2008), comparando a 
Tabela 1. Espécies de abelhas Euglossina capturadas por substância aromática em fragmento de floresta nas corredeiras do Bem-Querer, Caracaraí, Roraima.

\begin{tabular}{|c|c|c|c|c|}
\hline Espécie & $\begin{array}{c}\text { Óleo de } \\
\text { Eucalipto }\end{array}$ & $\begin{array}{c}\text { Salicilato de } \\
\text { metila }\end{array}$ & Eugenol & Total \\
\hline Eufriesea pulchra (Smith, 1854) & 2 & 0 & 0 & 2 \\
\hline Euglossa allosticta Moure, 1969 & 3 & 0 & 0 & 3 \\
\hline Eg. amazonica Dressler, 1982 & 3 & 0 & 0 & 3 \\
\hline Eg. analis Westwood, 1840 & 0 & 2 & 0 & 2 \\
\hline Eg. augaspis Dressler, 1982 & 2 & 1 & 11 & 14 \\
\hline Eg. chalybeata Friese, 1925 & 46 & 1 & 1 & 48 \\
\hline Eg. chlorina Dressler, 1982 & 2 & 0 & 0 & 2 \\
\hline Eg. cognata Moure, 1970 & 0 & 6 & 0 & 6 \\
\hline Eg. despecta Moure, 1968 & 1 & 0 & 0 & 1 \\
\hline Eg. ignita Smith, 1874 & 76 & 18 & 1 & 95 \\
\hline Eg. imperialis Cockerell, 1922 & 79 & 18 & 0 & 97 \\
\hline Eg. intersecta Latreille, 1838 & 22 & 0 & 0 & 22 \\
\hline Eg. ioprosopa Dressler, 1982 & 0 & 0 & 2 & 2 \\
\hline Eg. iopyrrha Dressler 1982 & 0 & 3 & 0 & 3 \\
\hline Eg. laevicincta Dressler, 1982 & 0 & 1 & 0 & 1 \\
\hline Eg. liopoda Dressler, 1982 & 1 & 0 & 1 & 2 \\
\hline Eg. mixta Friese, 1899 & 0 & 19 & 1 & 20 \\
\hline Eg. modestior Dressler, 1982 & 4 & 0 & 0 & 4 \\
\hline Eg. mourei Dressler, 1982 & 8 & 0 & 0 & 8 \\
\hline Eg. orellana Roubik, 2004 & 1 & 0 & 0 & 1 \\
\hline Eg. prasina Dressler, 1982 & 4 & 0 & 0 & 4 \\
\hline Eulaema bombiformis Packard, 1969 & 0 & 12 & 0 & 12 \\
\hline El. cingulata Fabricius, 1804 & 0 & 0 & 8 & 8 \\
\hline El. meriana Oliver, 1789 & 72 & 37 & 0 & 109 \\
\hline El. nigrita Lepeletier, 1841 & 3 & 0 & 0 & 3 \\
\hline Exaerete frontalis Guérin-Méneville, 1845 & 16 & 0 & 1 & 17 \\
\hline Ex. lepeletiere Oliveira \& Nemésio, 2003 & 1 & 1 & 1 & 3 \\
\hline Ex. smaragdina Guérin-Méneville, 1845 & 10 & 2 & 0 & 12 \\
\hline Total de espécies & 20 & 13 & 9 & 28 \\
\hline Total de espécimes machos & 356 & 121 & 27 & 504 \\
\hline
\end{tabular}

fauna de abelhas Euglossina em plantios de acácia australiana (Acacia mangium Willd) com floresta primária, os autores encontraram 21 espécies. Silva et al. (2013) comparando a diversidade de abelhas entre áreas de floresta alterada e primária na Serra do Tepequém encontraram 24 espécies de abelhas Euglossina.

Segundo Oliveira et al. (2010), a resposta para tantas espécies de Euglossina em um estado com uma superfície territorial pequena (cerca de $225.000 \mathrm{~km}^{2}$ ), possa ser explicada principalmente, pelo mosaico de ambientes encontrados em Roraima. De forma simplificada, a vegetação do Estado apresenta campos e cerrados na região centro-nordeste, florestas baixas a oeste e ao sul, florestas de altitude na fronteira norte e regiões de alagados no sul acompanhando as margens do rio Branco. Na Figura 02 os tipos vegetacionais de Roraima são apresentados com maior detalhamento juntamente com os locais dos estudos realizados com abelhas Euglossina em Roraima citados neste trabalho. Essa diversidade de ecossistemas gera um diversidade de plantas e flores influenciado, assim, a diversificação na fauna de abelhas Euglossina.

O gênero Euglossa foi o mais abundante 
Figura 2. Mapa da vegetação do estado de Roraima assinalando os locais de estudo. A. Silva (2002), B. Nemésio (2005), C. Maia e Silva (2008) e D. Silva et al. (2013).

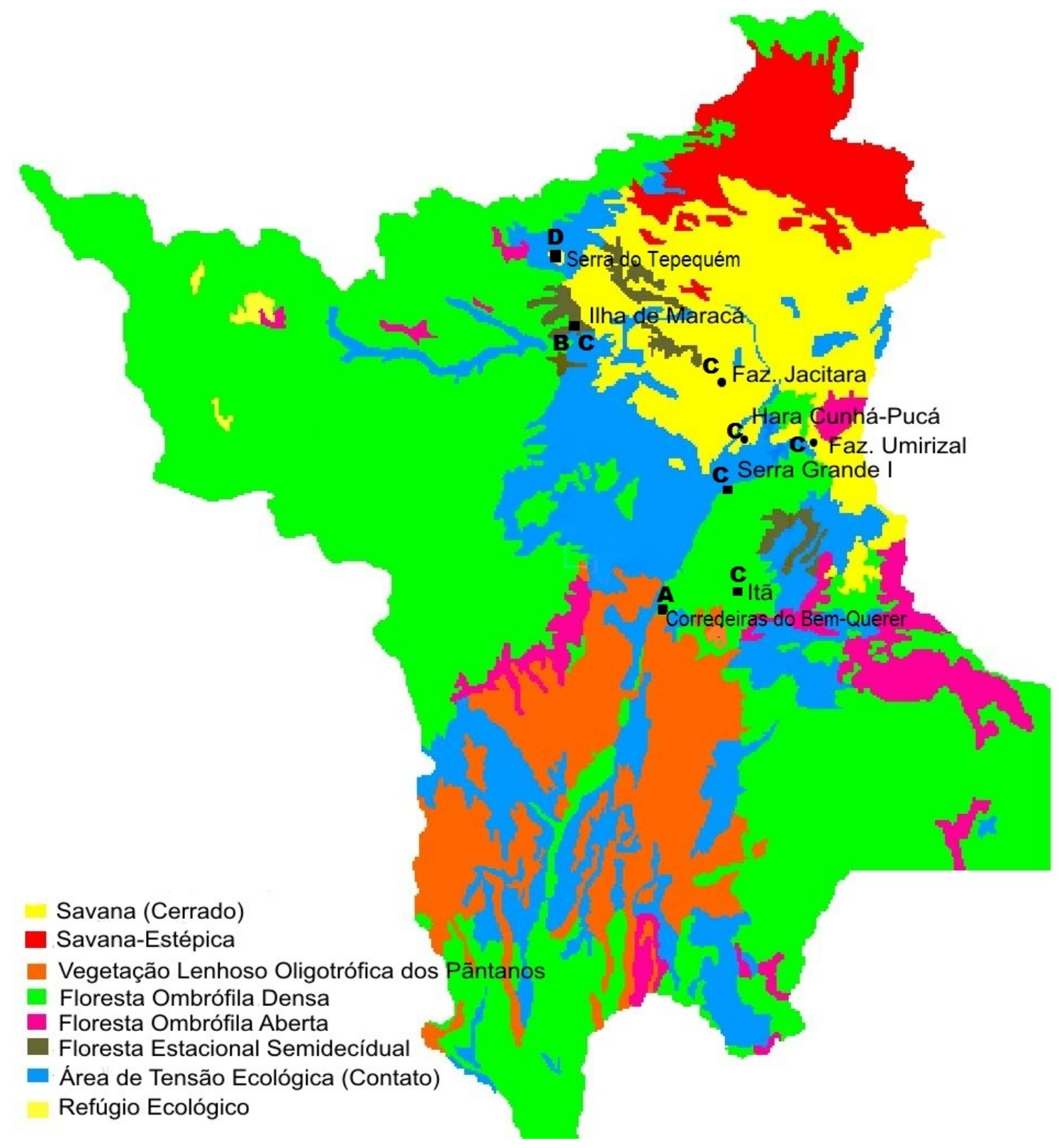

com 338 espécimes $(67,06 \%)$, seguido por Eulaema com 132 (26,19\%), Exaerete com 32 $(6,35 \%)$ e Eufriesea com dois espécimes $(0,40 \%)$ (Tabela 2).

A espécie Eulaema meriana Oliver, 1789 foi a mais abundante com 109 espécimes (21,62\%), seguida de Euglossa imperialis Cockerell, 1922 com 97 (19,24\%), Euglossa ignita Smith, 1874 com 95 indivíduos $(18,84 \%)$. As espécies Euglossa orellana Roubik, 2004, Euglossa despecta Moure, 1968 e Euglossa laevicincta Dressler, 1982, foram as de menor ocorrência com apenas um indivíduo cada (Tabela 1). Estes resultados são próximos aos obtidos por Oliveira et al. (2010), onde o gênero mais abundante foi Euglossa e diferiram de Maia \& Silva (2008), onde Eulaema foi o gênero mais abundante, em estudos realizados em Roraima.

A substância com maior atratividade foi o óleo de eucalipto com 356 indivíduos (70,35\%) e 20 espécies capturadas, seguida do salicilado de metila com 121 indivíduos $(23,91 \%)$ e 13 espécies e eugenol com 27 indivíduos (5,33\%) e nove espécies. As diferenças de atratividade entre as três substâncias utilizadas é estatisticamente significativa para o teste do X2 $(\mathrm{p}<0,0001)$, apontando uma maior preferência por óleo de eucalipto.

Esses resultados diferem de Storck-Tornon 
Tabela 2. Riqueza e abundância das abelhas Euglossina capturadas em fragmento de floresta nas corredeiras do BemQuerer, Caracaraí, Roraima.

\begin{tabular}{lcccc}
\hline Gênero & $\begin{array}{c}\text { Abundância } \\
\text { absoluta }\end{array}$ & $\begin{array}{c}\text { Abundância relativa } \\
\text { (\%) }\end{array}$ & Riqueza & Riqueza relativa (\%) \\
\hline Euglossa & 338 & $67,06 \%$ & 20 & $71,43 \%$ \\
Eulaema & 132 & $26,19 \%$ & 4 & $14,29 \%$ \\
Exaerete & 32 & $6,35 \%$ & 3 & $10,71 \%$ \\
Eufriesea & 2 & $0,40 \%$ & 1 & $3,57 \%$ \\
\hline Total & 504 & $100,00 \%$ & 28 & $100,00 \%$ \\
\hline
\end{tabular}

et al. (2009) onde, das seis substâncias aromáticas utilizadas pelos autores no Acre, o cineol atraiu maior número de indivíduos $(23,8 \%)$ seguidos por vanilina $(19,9 \%)$ e benzil acetato $(19,4 \%)$, enquanto que as substâncias aromáticas que atraíram maior número de espécies foram o salicilato de metila e o cineol. No estudo de Mendes et al. (2008), onde os autores utilizaram cinco substâncias aromáticas para atrair machos de Euglossina, estes também verificaram que óleo de eucalipto foi a substância mais atrativa e as menos efetivas foram a valina e o salicilato de metila.

A substância aromática eugenol, apesar de ter atraído menos exemplares, foi o único composto que pelo qual foram capturados indivíduos de Euglossa ioprosopa Dressler, 1982 e Eulaema cingulata Fabricius, 1804. Óleo de eucalipto foi exclusivamente atrativo para as espécies: Euglossa allosticta Moure, 1969, Euglossa amazonica Dressler, 1982, Euglossa chlorina Dressler, 1982, Euglossa despecta Moure, 1968, Euglossa intersecta Latreille, 1838, Euglossa modestior Dressler, 1982, Euglossa moure Dressler, 1982. As espécies: Euglossa orellana Roubik, 2004, Euglossa prasina Dressler, 1982, Eulaema nigrita Lepeletier, 1841, Eufriesea pulchra (Smith, 1854), só foram capturadas com óleo de eucalipto. As espécies: Euglossa cognata Moure, 1970, Euglossa analis Westwood, 1840, Euglossa laevicincta Dressler, 1982, Eulaema bombiformis Packard, 1969 só foram capturadas utilizando armadilhas contendo salicilato de metila.

\section{CONCLUSÕES}

Foram capturados 510 espécimes de abelhas Euglossina, destas 504 foram identificados ao nível de espécie. O total de espécies capturadas foi de 28 sendo, 20 do gênero Euglossa, quatro do gênero Eulaema, três do gênero Exaerete e uma do gênero Eufriesea e nenhum indivíduo do gênero Aglae foi capturado.

A substância que possibilitou a captura do maior número de indivíduos e espécies foi $\mathrm{o}$ óleo de eucalipto.

Nesse trabalho são registradas três novas ocorrências de espécies de abelhas Euglossina para as corredeiras do Bem-Querer que são: Euglossa ioprosopa Dressler, 1982, Euglossa orellana Roubik, 2004, Exaerete lepeletiere Oliveira \& Nemésio, 2003.

\section{REFERENCIAS BIBLIOGRÁFICAS}

Aguiar, W.M.A. 2006. Comunidade de abelhas Euglossina (Hymenoptera, Apidae) em remanescentes de mata estacional semidecidual sobre tabuleiro no estado do Rio de Janeiro. Dissertação de Mestrado. Universidade Estadual do Norte Fluminense Darcy Ribeiro, 62p.

Becker, P.; Moure, J.S. \& Peralta, F.J.A. 1991. More about Euglossine bees in Amazonian Forest fragments. Biotropica 23: 586-591.

Crane, E. 1972. Bees in the pollination of seed crops. Journal of the R.A.S.E. 133: 119-135.

Janzen, D.H.1971. Euglossine bees as long-distance pollinators of tropical plants. Science 171: 203-205.

Maia, T.F.S. \& Silva, R.J.S. 2008. Análise faunística de abelhas Euglossina (Hymenoptera: Apidae) em ambientes de floresta nativa e plantios de Acacia mangium no Estado de Roraima. Revista Agro@mbienteonline 2(2): 42-50.

Mendes, N.F.; Rêgo, M.C.M.; Carvalho, C. C. 2008. Abelhas Euglossina (Hymenoptera, Apidae) coletadas em uma monocultura de eucalipto circundada por Cerrado Urbano, Santos, Maranhão, Iheringia, Série. Zoológica 98(3): 285-290.

Morato, F.E. \& Alencar, P.J.B. 2009. Efeitos de borda sobre as assembleias de abelhas das orquídeas (Hymenoptera: Apidae: Euglossina), Acre, Brasil. 
Anais do IX Congresso de Ecologia do Brasil, São Lourenço - MG.

Moure, J.S., Urban, D. \& Melo, G.A.R. (Orgs). Catalogue of Bees (Hymenoptera, Apoidea) in the Neotropical Region - online version. ( http:// www.moure.cria.org.br/catalogue). Acesso em 16/02/2015.

Nemésio, A. 2005. Orchid bees (Hymenoptera: Apidae) of ilha de Maracá, Roraima, Northem Brazil. Lundiana 6(2): 117-119.

Nemésio, A. 2013. The orchid-bee fauna (Hymenoptera: Apidae) of 'Parque Nacional do Monte Pascoal', 'Parque Nacional do Descobrimento' and three other Atlantic Forest remnants in southern Bahia, eastern Brazil. Brazilian Journal of Biology 73 (2): 437-446, 2013.

Oliveira, L.M.; Silva, R.J.S.; Silva. C.M; Araújo, O.C.A; Albuquerque, C.I.M. \& Tavares, F.S. 2010. Abelhas de Roraima: porque tantas espécies em tão pouco espaço. In: Barbosa, R. I; Melo, V. F. Roraima Homem Ambiente Ecologia. Femact, Boa vista, p.523-540.

Peruquetti R.C. 2000. Function of fragrances collected by Euglossini males (Hymenoptera: Apidae). Entomologia Generalis 25: 33-37.

Roubik, D.W. 1989. Ecology and natural history of tropical bees. Cambridge Univ Press, New York, $514 p$.

Silva, M.C. 2002. Levantamento da fauna de abelhas Euglossinae (Hymenoptera, Apidae), em floresta primária, na área das corredeiras do Bem-Querer, município de Caracaraí, estado de Roraima, Brasil. Monografia. Universidade federal de Roraima, Boa Vista, 61p.

Silva, S.J.R. 2010. A produção de mel em plantios de Acacia mangium Willd. In: Tonini, H.; HalfeldVieira, B.A.; Silva, S.J.R. Acacia mangium características e seu cultivo em Roraima. 1. ed. Boa vista, RR: Embrapa Roraima, p.133-145.

Silva, S.J.R, Gama Neto, J.L. \& Parente, F.S. 2013. Abundância e diversidade de abelhas Euglossina (Hymenoptera: Apidae) da Serra do Tepequém, município de Amajari, Roraima, Brasil. Revista Agro@ambiente online 7(2): 225-230.

Silveira, F.A.; Melo, G.A.R,; Almeida, E.A.B. 2002. Abelha brasileiras: Sistemática e identificação: Belo Horizonte, $253 \mathrm{p}$.

Storck-Tornon, D.; Morato, E.F..; Oliveira, M.L. 2009. Fauna de Euglossinae (Hymenoptera: Apidae) da Amazônia sul-ocidental, Acre, Brasil. Acta Amazônica 39(3): 693-706.

Tales, A.; Gonçalves, V.; Elder, F. \& Pollyanna, J.B.A. 2009. Efeitos de borda sobre as assembleias de abelhas das orquídeas (Hymenoptera: Apidae: Euglossina). Anais do IX Congresso de Ecologia do
Brasil, 13 a 17 de Setembro de 2009. São Lourenço, MG.

Wikelski, M.; Moxley, J.; Eaton-Mordas, A.; LópezUribe, M.M.; Holland, R.; Moskowitz, D.; Roubik, D.W. \& Kays, R. 2010. Large-range movements of Neotropical orchid bees observed via radio telemetry. Plos One 5: 1-6. 